\title{
Emergence of simple patterns in many-body systems: from macroscopic objects to the atomic nucleus
}

\author{
R.F. Garcia Ruiz ${ }^{1,2}$ and A. Vernon ${ }^{3}$ \\ 1 CERN, CH-1211 Geneva 23, Switzerland \\ 2 Massachusetts Institute of Technology, Cambridge, MA 02139, USA \\ 3 KU Leuven, Instituut voor Kern- en Stralingsfysica, B-3001 Leuven, Belgium
}

Received: date / Revised version: date

\begin{abstract}
Strongly correlated many-body systems often display the emergence of simple patterns and regular behaviour of their global properties. Phenomena such as clusterization, collective motion and appearance of shell structures are commonly observed across different size, time, and energy scales in our universe. Although at the microscopic level their individual parts are described by complex interactions, the collective behaviour of these systems can exhibit strikingly regular patterns. This contribution provides an overview of the experimental signatures that are commonly used to identify the emergence of shell structures and collective phenomena in distinct physical systems. Examples in macroscopic systems are presented alongside features observed in atomic nuclei. The discussion is focused on the experimental trends observed for exotic nuclei in the vicinity of nuclear closed-shells, and the new challenges that recent experiments have posed in our understanding of emergent phenomena in nuclei.
\end{abstract}

PACS. PACS-key discribing text of that key - PACS-key discribing text of that key

\section{Introduction}

Our understanding of the universe is intimately related to our description of many-body systems. The knowledge of the fundamental particles and forces of nature is as important as our ability to understand how these building blocks are organized to form complex systems. Remarkably, the emergence of simple and regular patterns are common features observed in strongly-correlated manybody systems [1-9]. At the microscopic level, the individual parts of different physical systems can be described by fundamentally different interactions, however, their collective behaviour can exhibit similar patterns. These seemingly simple regularities of certain properties of a physical system tends to suggest the existence of underlying symmetries and allows simple models to provide a good description of the observed data [9-13]. However, the link between these models and the underlying microscopic interactions is an open question in many fields of physics.

The recent progress of both experimental and theoretical developments have allowed an unprecedented connection between reductionist and emergent views of nature. Advances in many-body methods and the rapid development of computing power have provided new paths towards the ab-initio description of macroscopic phenomena. Theoretical developments are motivated by the ambition of a first principles description of emergent phenomena, yet this reductionist approach is deeply motivated by empirical observations $[14,15]$. The emergence of unexpected phenomena is uniquely accessed through experiments. A deeper understanding of the microscopic origin of the observed physical phenomena is achieved through systematic experimental studies confronted with the theoretical descriptions.

This article presents a short overview of experimental signatures that are commonly used to characterize the emergence of phenomena in different physical systems. Various examples of objects from the human size scale down to the fentometer scale are presented. The discussion is centered on the observables that are used to indicate the emergence of phenomena such as shell structures and "magic" numbers - integer number of constituents with notably different properties. Albeit not exhaustive, an effort is made to include citations that could be useful to direct interested readers to the relevant literature.

The manuscript is divided in two main parts: The first part provides a brief description of selected examples that illustrate the emergence of regular patters in macroscopic systems and their relation with similar patterns observed in the atomic nucleus. The second part is focused on the experimental signatures used to discuss the emergence of collective phenomena and shell structures in nuclei. Commonly discussed properties such as binding energies, nuclear charge radii, excitation energies and transition probabilities are presented. The discussion is expanded on recent experimental results obtained for the ground-state properties of nuclei in the neighborhood of nuclear shell 
closures. Finally, an emphasis is made on the trends and open questions that the new observations pose for our current understanding of nuclear structure at different regions of the nuclear chart.

\section{Emergence of simple patterns in many-body systems}

Throughout nature, driving forces give rise to optimisation problems for the arrangement of constituents in manybody systems at almost every size scale $[1-5,7,16,17,21$, $22,24-27,29,41-45]$. On biological scales we can see this manifests in a variety of collective phenomena and pattern formation $[1,46-49]$. Such as in the phyllotaxis of plants $[44,50,51]$, where simply describable patterns of growth appear for the arrangement of leaves or flowers around a plant stem. One particularly striking example is in the growth of seeds in a sunflower head $[16,17]$, in which the number of spirals of seeds follows the Fibonacci sequence, a natural result of optimised packing despite the large number of constituents seeds. A large variety of patterns emerge in smaller systems as a consequence of the optimal arrangement of their constituents $[46,47,52]$, from the clustering in framboidal pyrite $[53,54]$ to the crowding of molecules in cells $[43,55,56]$, or that of DNA strands in cell nuclei $[42,57]$. Complex many-body systems often form clusters to minimise their energy by interactions with their neighbours and their mean field. This situation can give rise to "magic" numbers, as with those in the atomic nucleus, where certain integer numbers of constituents of a given system results in greater stability of its collective whole. The simplest signature for these magic numbers is the greater abundance of systems with the magic number of constituents compared to those with the less stable configurations of with other numbers of constituents [7,21-24,24-26]. This is comparable with the natural abundance distribution of isotopes in the universe $[58,59]$ following nucleosynthesis [60]. The energetic efficiency of these more stable magic number configurations leads to their preponderance in biological systems also [61-64], such as with the magic number of capsid proteins which appear to form virus capsid structures [18]. A summary of different systems in nature which exhibit these magic numbers of stability is shown in Table 1 . The experimental signatures and typical size of the different systems are shown in the same table. For nondeformable constituents with their packing constrained by symmetric polyhedral shapes, the magic numbers that appear can be determined for a system with constituents of any size using purely geometric considerations, and they appear in nature with these numbers when this is the case $[1,24,29,65-67]$. However the identification of naturally occuring magic numbers from a distribution becomes more challenging for greater length scales, as the likelyhood of finding ensambles with a similar number of homogenous constituents decreases.

Those constituents between magic numbers, can also be said to belong to a 'shell', in analogy to the electronic shells of atoms [30] or for nucleons in atomic nuclei $[68,69]$. In some cases this is reflected by the spatial arrangement of the constituents. This has been observed for example in 'dusty' plasmas [70,71], where charged dust particles (on the micrometer scale) can self assemble into a plasma crystal arrangement with a radial spherical shell distribution of particles $[3,72]$, with the total system on the scale of millimeters. Such mesoscopic systems are often called 'artificial atoms' due to their close similarity with atomic systems. The examples enlisted in Table 1 for dust particles, occur for particular experimental conditions. These experiments have several highly tunable parameters, which can result in different sequences of magic numbers [72-74]. Similar self arrangement and appearance of magic numbers has also been observed in 2-dimensional mesoscopic experiments using micrometer sized superconducting disks $[4,75]$.

At the scale of hundreds of micrometers, polystyrene spheres with diameters of around $200 \mathrm{~nm}$ have been observed to form clusters of colloidal particles [76,77], which can be arranged in many configurations and also exhibit magic numbers, where specific numbers of colloidal particles were found to result in higher thermodynamic stability, as observed in decreased evaporation rates [5]. Similar to that of the total binding of an unstable nucleus as reflected in its half-life [31-33]. Due to the absence of a repulsive force these colloidal systems can range from a few particles [78] to billions of particles (colloidal crystals) $[79,80]$

Perhaps the systems with the most in common with the atomic nucleus are atomic clusters $[9,81,82]$, an area of physics which has historically benefited from analogies with nuclear models [82-84]. Clusters of atoms were observed to have magic numbers of enhanced stability reflected in their produced mass abundance spectra [24-26, 85] (see Table 1). The electronic structure of the constituent atom ultimately dictates the properties of the atomic clusters, however phenomenological models have been developed to provide a good description of the observed magic numbers, similar to the shell model of the atomic nucleus $[8,86]$. A 'wine-bottle' shaped potential used to describe these atomic clusters was adapted from the Woods-Saxon potential [85]. This potential predicted 'super shells' to appear as the number of atoms in the clusters approaches $N=1000$ [84], due to higher-order stabilising effects, analogous to the predicted islands of stability of heavy nuclei [87]. The predicted super-shell magic numbers were soon observed in sodium clusters [27]. Deformation also has an analogous role in these clusters as in atomic nuclei, where the most stable clusters have spherical deformation and those between shell closures have oblate or prolate deformation $[8,88]$, the Nilsson model of the atomic nucleus [89] has been adapted to describe axially deformed clusters, known as the Clemenger-Nilsson model [90]. Giant dipole resonances of atomic nuclei [91] also have a counterpart in these cluster systems, in the form of plasma resonance frequencies [92, 93]. Taking the example of the sodium clusters, many of the observables corroborate the same set of magic numbers [26, 28, 94-96] 
Table 1. Experimental signatures of the emergence of shell structures and "magic" numbers in different many-body systems. The size scales and the common observables that are used to characterize the properties of each systems are indicated. Here the ellipses ("...") are used to denote that additional magic numbers have been omitted for space.

\begin{tabular}{|c|c|c|c|c|c|c|}
\hline Constituent & Size & System & Size & Observable(s) & Magic numbers & Refs. \\
\hline Spheres & Any & $\begin{array}{l}\text { Spherical } \\
\text { confinement }\end{array}$ & Any & Density & $6,12,21,25,38 \ldots$ & {$[1]$} \\
\hline Sunflower seeds & $\stackrel{\sim}{1 \mathrm{~cm}}$ & $\begin{array}{l}\text { Sunflower } \\
\text { head }\end{array}$ & $5-50 \mathrm{~cm}$ & Number of spirals & $3,5,8,13,21,34, \ldots$ & {$[2,16,17]$} \\
\hline Dust particles & $\mu \mathrm{m}$ & $\begin{array}{l}3 \text { D plasma } \\
\text { crystal }\end{array}$ & $\mathrm{mm}$ & Radial distribution & $2,21,60,107 .$. & {$[3]$} \\
\hline $\begin{array}{l}\text { Superconducting } \\
\text { disks }\end{array}$ & $\mu \mathrm{m}$ & Vortex shells & $5 \mu \mathrm{m}$ & Radial distribution & $5,16,32$ & {$[4]$} \\
\hline $\begin{array}{l}\text { Polystyrene } \\
\text { spheres }\end{array}$ & $\begin{array}{l}244 \\
\mathrm{~nm}\end{array}$ & Colloidal cluster & $\sim 2.8 \mu \mathrm{m}$ & Evaporation rate & $\begin{array}{l}135,297,851,801 \\
1283,2583 \ldots\end{array}$ & {$[5]$} \\
\hline $\begin{array}{l}\text { Spherical } \\
\text { capsid protein }\end{array}$ & $\begin{array}{l}\sim 5 \\
\mathrm{~nm}\end{array}$ & $\begin{array}{l}\text { Virus } \\
\text { capsid structure }\end{array}$ & $\sim 50 \mathrm{~nm}$ & Abundance & $15,17,18,42$ & {$[18-20]$} \\
\hline C atoms & $\begin{array}{l}170 \\
\mathrm{pm}\end{array}$ & Fullerenes & $\sim 6 \mathrm{~nm}$ & Mass abundance & $\begin{array}{l}60,70,72,76,78,84 \\
. .\end{array}$ & {$[7,21,22]$} \\
\hline $\mathrm{H}_{2} \mathrm{O}$ & $\begin{array}{l}275 \\
\mathrm{pm}\end{array}$ & $\begin{array}{l}\text { Electron-bound } \\
\text { water clusters }\end{array}$ & $\sim 3-20 \AA$ & Mass abundance & $2,6,7,11$ & {$[23]$} \\
\hline Xe atoms & $\begin{array}{l}216 \\
\mathrm{pm}\end{array}$ & Atom clusters & $\sim 2-10 \AA$ & Mass abundance & $\begin{array}{l}13,16,19,25,55,71 \\
87,147 \ldots\end{array}$ & {$[24,25]$} \\
\hline \multirow[t]{2}{*}{$\mathrm{Na}$ atoms } & $\begin{array}{l}227 \\
\mathrm{pm}\end{array}$ & Atom clusters & $\sim 2-10 \AA$ & $\begin{array}{l}\text { Mass abundance } \\
\text { Ionization energy }\end{array}$ & $8,20,40,58,92 \ldots$ & {$[26-28]$} \\
\hline & & & & Melting temperature & $55,116,147,178 \ldots$ & {$[29]$} \\
\hline Electrons & $\mathrm{fm}$ & Atoms & $31-348 \mathrm{fm}$ & Ionization energy & $2,10,18,36,54$ & {$[30]$} \\
\hline Nucleons & $\mathrm{fm}$ & Nuclei & $1-10 \mathrm{fm}$ & $\begin{array}{l}\text { Binding energies, } \mathrm{t}_{1 / 2} \\
<r^{2}> \\
\mathrm{B}(\mathrm{E} 2), \mathrm{E}_{2+} \\
\mathrm{Q}_{S}, \mu \\
\text { Solar abundances } \\
\text { Neutron capture } \sigma \mathrm{s}\end{array}$ & $2,8,20,28,50,126 \ldots$ & $\begin{array}{l}{[31-35]} \\
{[36]} \\
{[37]} \\
{[38]} \\
{[6,39]} \\
{[35,40]}\end{array}$ \\
\hline
\end{tabular}


which are of electronic origin. A modified set of magic numbers was found in the melting temperatures of the clusters [97]. However, this required an additional interpretation considering the geometric shells of the positions of the atomic nuclei alongside the electronic shells, due to the importance of the positions of the atomic nuclei in the melting process [29]. This highlights how experimental observables can probe very different aspects of the same physical system, leading to different sequences of magic numbers for different properties of the same system.

\section{Global trends and simple patterns in nuclei}

The atomic nucleus provides a rich laboratory in the studies of strongly correlated many-body systems. Due to the high nuclear density and the short- and long-range properties of the nuclear force, nuclei are highly sensitive to twoand higher-order many-body forces. The non-perturbative character of the strong force requires highly demanding theoretical treatments. In contrast to the electromagnetic forces, three-body forces appear at a fundamental level in the strong interaction [98]. By varying the numbers of protons and neutrons in the nucleus, inter-nucleon correlations can drive very different collective phenomena [99-102]. Intriguingly, a set of regular patterns appear across the whole nuclear chart [103-108]. These seemingly simple patterns have motivated numerous phenomenological models since the early days of nuclear physics. Simple model principles such as independent-particle motion $[109,110]$ and the semi-classical collective motion of nuclei [111] have been very successful in providing a global description of the observed nuclear phenomena.

Below we present a short overview of the experimental signatures of nuclear shell structures and collective phenomena that are commonly discussed in literature. While similar signatures and correlations are found in different observables, some of these correlations appear to break down in neutron-rich systems, where different patterns emerge. This discussion is expanded upon using the evolution of nuclear properties in the neighborhood of the neutron-rich ${ }^{52} \mathrm{Ca}(Z=20),{ }^{78} \mathrm{Ni}(Z=28)$, and ${ }^{132} \mathrm{Sn}$ $(Z=50)$ isotopes as examples, where recent theoretical and experimental results are available.

\subsection{Experimental signatures of shell structures}

The signatures of nuclear shell structures are manifested in different observables $[101,105,112-115]$. The number of nucleons that completely fills nuclear closed-shells are the so-called "magic" numbers. Nuclei with a magic number of nucleons are commonly observed to have the following experimental signatures: i. a relatively small root-meansquared charge radius, $\left\langle r^{2}\right\rangle$. This is seen as a pronounced change of the charge radius after a shell closure ("kink"), with a smooth increase towards shell closures, and a larger increase through the filling of the new open shell [106]. ii. a relatively large two-nucleon separation energy, $S_{2 n}$; iii. a small quadrupole moment value, $Q_{s}$; iv. a high excitation energy of the first $2^{+}$state, $E_{2+}$; and v. a small transition probability to the first $2^{+}$excited state, $B(E 2)$. A compilation of these experimental properties as a function of the neutron and proton numbers are shown in Figure 1 and Figure 2, respectively. The data corresponding to different isotones are shown in Figure 1, using bars of different colors to indicate the magnitudes of the observables for each isotone, the same is shown in 2 as a function of atomic number.

The changes of the root-mean square charge radii when two neutrons are added, $\Delta\left\langle r^{2}\right\rangle(2 \mathrm{n})$, are presented in Figure 1 . The analogous differences when two protons are added, $\Delta\left\langle r^{2}\right\rangle(2 \mathrm{p})$, are shown in Figure 2, however the data in this case is relatively sparse as the charge radii of many elements have not yet been measured. At magic number of nucleons these differences exhibit a minimum value, with local maximum after crossing the closed shell. As the magnitude of $Q_{s}, B(E 2)$, and $E_{2+}$ scales with the atomic number and the nuclear size, these parameters were normalized in order to compare light and heavy nuclei on the same scale. The experimental values of $Q_{s}$ and $B(E 2)$ were scaled to the dimensionless values $Q_{s} / Z R$ and $B(E 2) / Z^{2} R^{2}$, with $Z$ the proton number and $R=$ $1.18 A^{1 / 3}$ the drop-model radius. Normalized observables present minimum values around the nucleon numbers 28 , 50,82 and 126, with a clear correlation seen in the trends of all observables. For some isotopes, additional local minima appear around nuclear numbers 2, 8, 16, 20, and 40. Figure 1 iv), for example, shows bars of different color at $N=20$, indicating that nuclei with the same number of neutrons, such as ${ }^{32} \mathrm{Mg}$ and ${ }^{40} \mathrm{Ca}$, have very different $E\left(2^{+}\right)$values [141]. The isotopes with magic nucleon numbers have relatively high binding energy, and their charge distribution exhibit smaller variations with respect to the spherical shapes (small quadrupole moments). The nuclear charge radius commonly increases with the number of nucleons, but the slope of the increase is notably smaller approaching the nuclear closed shells. These nuclei are more difficult to excite than their neighbors, which is evidenced by their relatively high excitation energies and low excitation probabilities.

The properties of light nuclei $(A<20)$ exhibit different patterns with respect to the number of nucleons. These nuclei do not exhibit the regular trends observed in heavier isotopes. The addition or removal of a single nucleon can produce drastic changes on the properties of these fewnucleon systems. Common patterns are more evident in heavy nuclei, with a few points outside the general trends. Some particular isotopes, as in the region around $Z=40$, $N=60$ and $Z=62, N=90$, are considered to present a rapid onset of deformation [99, 142]. Interestingly, collective phenomena such as shape coexistence and phase transitions observed for nuclei in the region $Z=62, N=90$ have been suggested to exhibit analogous features as those for silicon clusters, which are governed by very different interactions [143]. 


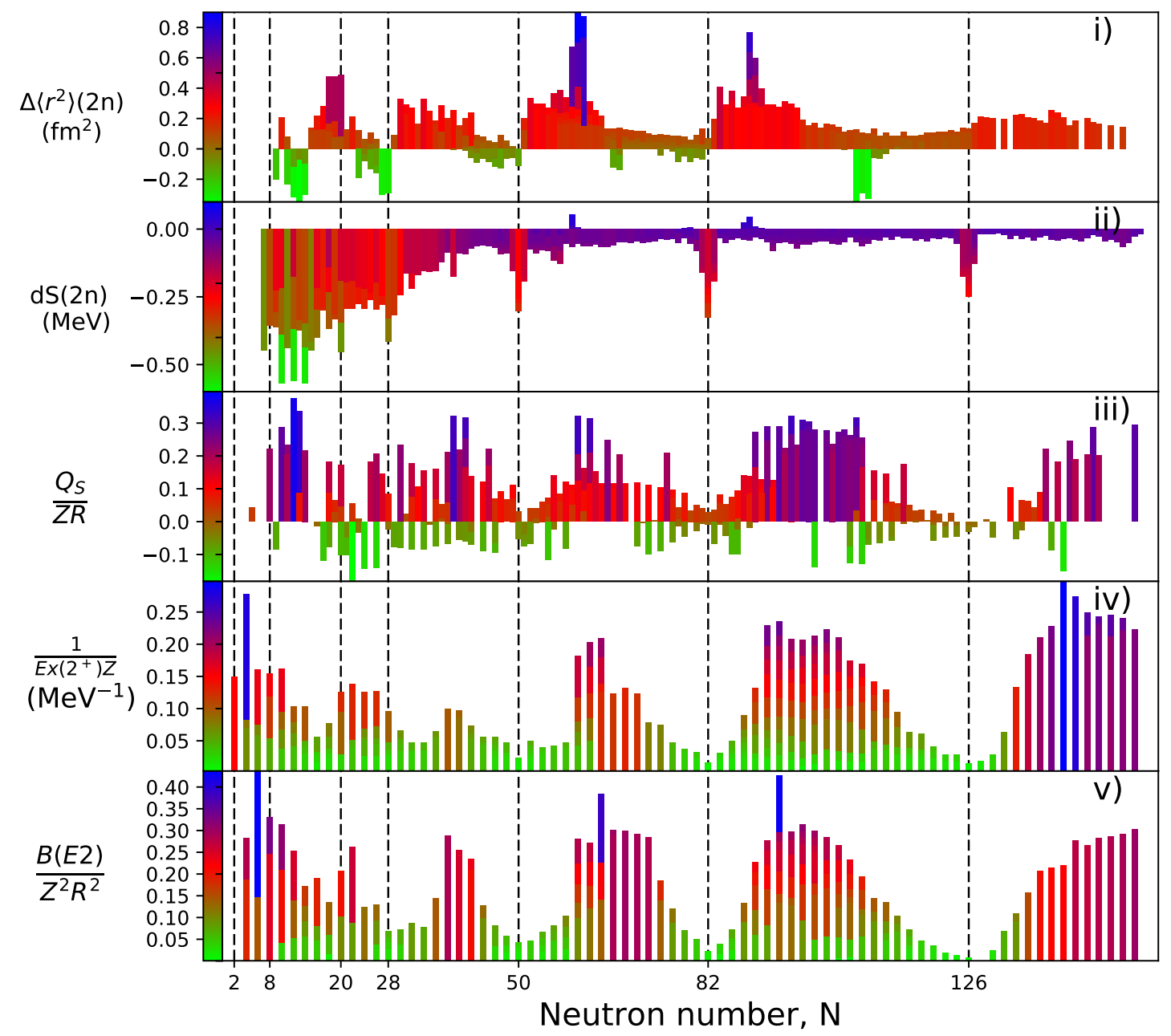

Fig. 1. Experimental nuclear properties as a function of the neutron number: i. root-mean-squared charge radii difference when two neutrons are added, $\left\langle r^{2}\right\rangle(2 \mathrm{n})$; ii. derivative of the two-neutron separation energy $\mathrm{d} S_{2 n}$; iii. normalized spectroscopic quadrupole moments $Q_{s} / Z R$; iv. scaled inverse of the excitation energy of the first $2^{+}$state, $1 / E_{2} Z$; and v. normalized transition probability to the first $2^{+}$excited state, $B(E 2) / Z^{2} R^{2}$. Data taken from $[31-33,36-38,101,113,116-140]$.

\subsection{Simple patterns in complex nuclei}

Nuclear electromagnetic moments such as the magnetic dipole and electric quadrupole provide complementary insights into the microscopic and collective properties of nuclei $[144,145]$. In fact, electromagnetic moments played a key role in motivating the most basic models of nuclear physics: the nuclear shell model [109], and nuclear deformation [146-148]. Systematic experimental studies of isotopes around nuclear closed shells have revealed surprisingly simple trends in the evolution of nuclear groundstate electromagnetic properties as a function of the neutron number [113,116-118,144,149-151].

Nuclei in the vicinity of the tin isotopes give outstanding examples of simple patterns. The electromagnetic properties of these complex nuclei, with around 50 protons and more than 50 neutrons, seem to be described by a single particle in a nuclear orbital. The experimental nuclear $g$-factor (the ratio between the dipole magnetic moment and the nuclear spin) and electric quadrupole moments of cadmium $(Z=48)$, indium $(Z=49)$, and tin $(Z=50)$ isotopes are shown in Figure 4, exhibiting simple trends as a function of neutron number. A simplified single-particle model provides a good description of these observations. In the shell model picture, the electromagnetic properties of odd-even indium isotopes are given by a single proton hole in the $\pi h_{11 / 2}$ orbit [152-154]. This simple picture of nuclear structure seems to be supported by a rather constant value of their nuclear moments, which present very small variations when neutrons are added. For the evenproton nuclei, cadmium and tin, the naive shell-model expectation is that that the electromagnetic properties of even-odd isotopes are dominated by a single neutron occupying the $\nu h_{11 / 2}$ neutron orbit. This idea is also supported by a constant value of the magnetic moment, and 


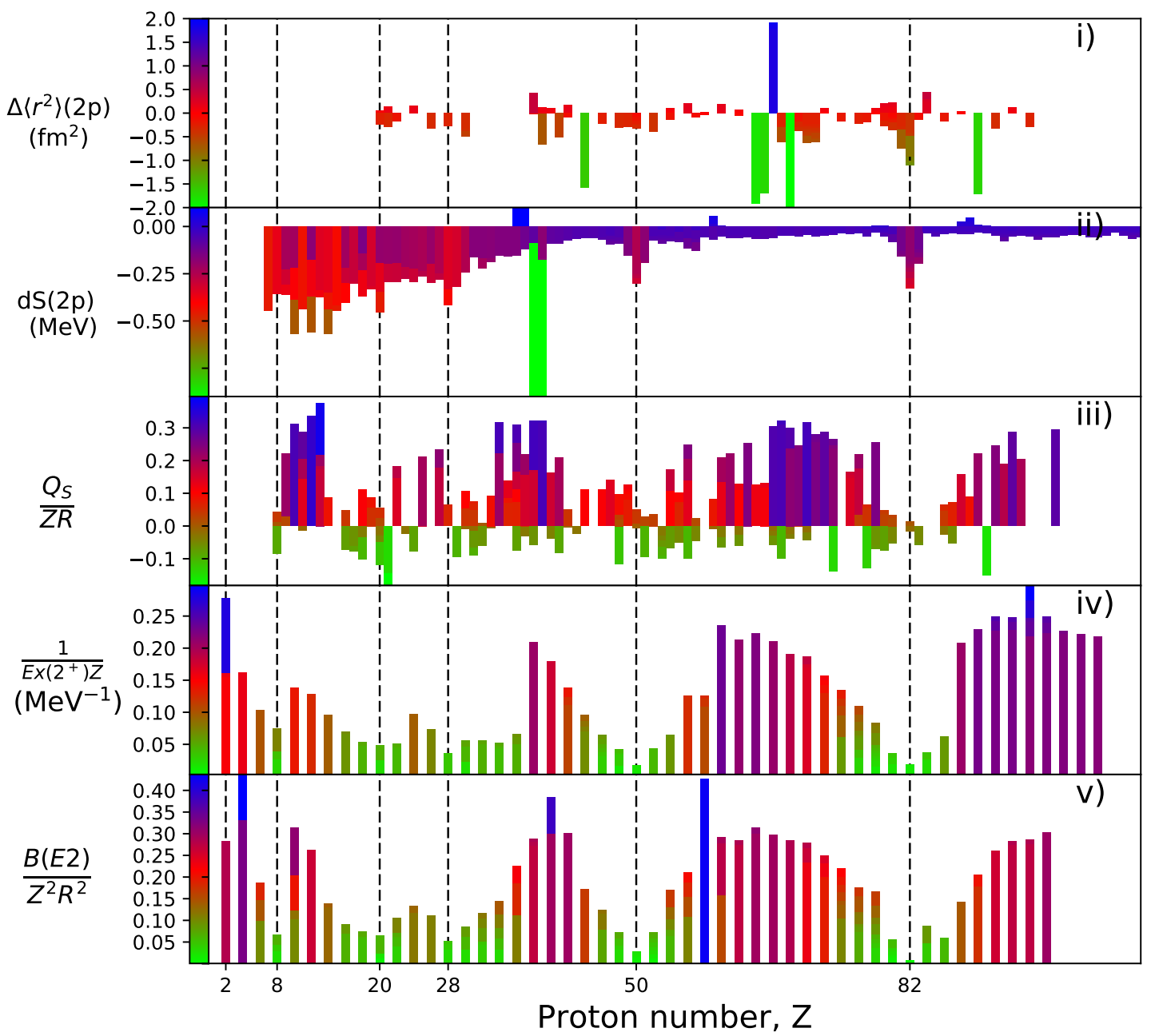

Fig. 2. Experimental nuclear properties as a function of the proton number: i. root-mean-squared charge radii difference when two protons are added, $\left\langle r^{2}\right\rangle(2 \mathrm{p})$; ii. derivative of the two-proton separation energy $\mathrm{d} S_{2 n}$; iii. normalized spectroscopic quadrupole moments $Q_{s} / Z R$; iv. scaled inverse of the excitation energy of the first $2^{+}$state, $1 / E_{2} Z$; and v. normalized transition probability to the first $2^{+}$excited state, $B(E 2) / Z^{2} R^{2}$. Data taken from $[31-33,36-38,101,113,116-140]$.

a linear trend in the nuclear quadrupole moments. In this shell model picture, a particle occupying an orbit around closed shells has a negative quadrupole moment, which is interpreted as polarizing a spherical core towards an oblate deformation $\left(Q_{s}<0\right)$ [144]. If neutrons are added to the same orbit, the values of quadrupole moments cross zero when the orbit is half-filled, and take positive values when more than half of the orbit is occupied. This is interpreted as a "hole" polarizing the core towards prolate deformation $\left(Q_{s}>0\right)$. Similar trends have been observed in the calcium $(Z=20)[113]$, nickel $(Z=28)[155]$ and lead $(Z=82)[144]$ regions.

\subsection{New trends in neutron-rich nuclei}

Recent developments in both experimental and theoretical tools have provided a deeper insight in our understand- ing of nuclear properties at extreme proton-to-neutron ratios. Particular interest has been focused on the evolution of nuclear properties towards the suggested neutron-rich doubly-magic nuclei: ${ }^{52,54} \mathrm{Ca}(Z=20, N=32,34)[101$, $114,115,121],{ }^{78} \mathrm{Ni}(Z=28, N=50)[123,124,156,157]$, and ${ }^{132} \mathrm{Sn}(Z=50, N=82)[127,128]$. These regions of the nuclear chart are being studied by several experimental techniques proving tests of theoretical descriptions at limits of the nuclear existence. While most of the measured experimental properties $\left(S_{2 n}, E\left(2^{+}\right), B(E 2)\right.$, and $Q_{s}$ ) have been described by available nuclear models $[113-115,157,158]$, the description of the nuclear size $\left(\left\langle r^{2}\right\rangle\right)$ has posed new challenges for modern nuclear theory $[101,128,129,159,160]$. This problem has been addressed with density functional theory, where satisfactory description of charge radii have been obtained in the calcium [129] 
i)
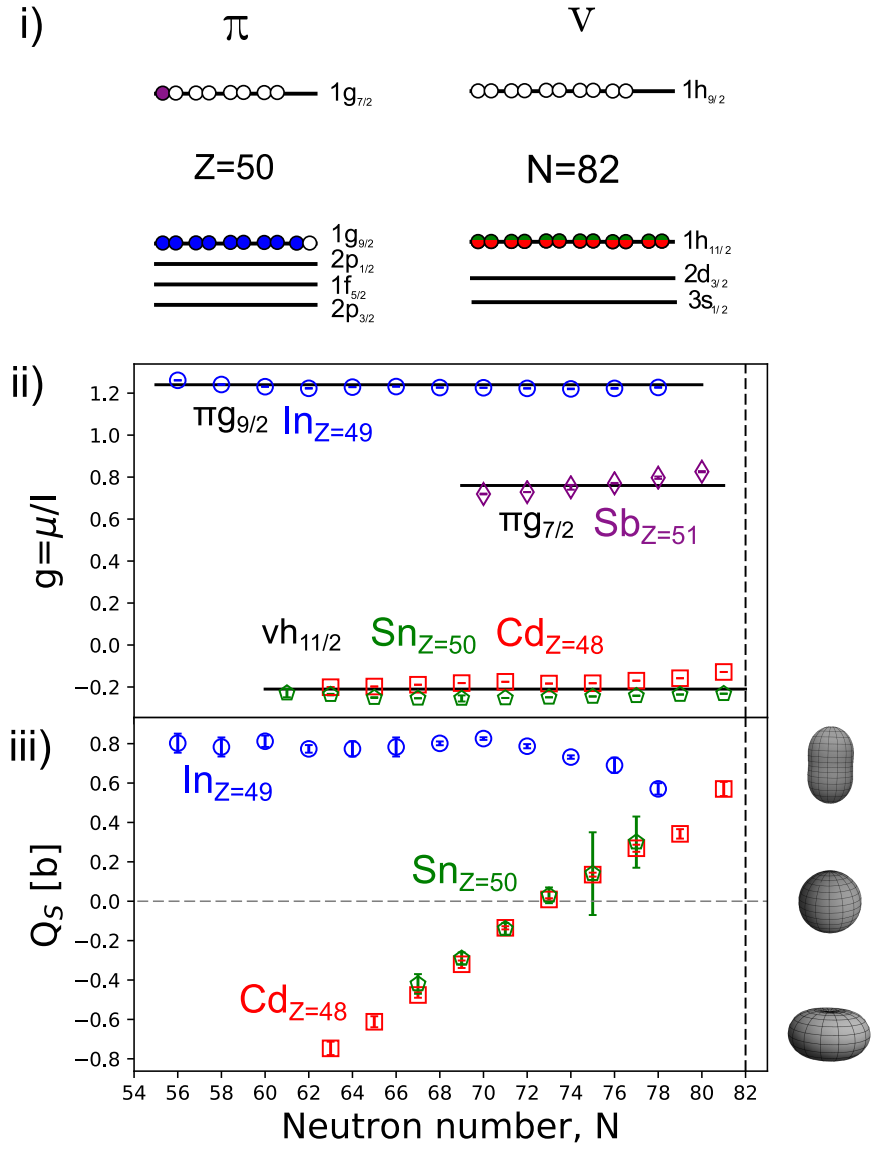

Fig. 3. Nuclear $g$-factor (i) and quadrupole moments (ii) of cadmium $(Z=48)$, indium $(Z=49)$, and tin $(Z=50)$ isotopes. The relevant nuclear shell model orbits are shown on the upper panel. Experimental data were taken from [38,116-120]

and tin regions [128]. However, a description in the abinitio framework has not been achieved yet $[101,160]$.

Figure 4 shows the changes of the root-mean-squared charge radii around the calcium, nickel, and tin regions. The values for each isotopic chain are shown with respect to the value at the closed neutron shells. While a strong element dependence is seen close to stability, the charge radii of neutron-rich isotopes beyond the neutron closedshell appear to increase with surprisingly similar slopes. The radii of the proton closed-shell calcium isotopes increase as rapid as the open shell isotopes $\mathrm{Mn}(Z=25)$ and $\mathrm{Fe}(Z=26)$.

Similar charge radii trends have been observed for isotopes around the nickel and tin regions. As illustrated in Figure 4 ii), the nuclear charge radii evolution in the nickel region present a noticeable dependence with the atomic number up to the neutron number $N=50$. However, for neutron-rich nuclei the root-mean-squared charge radii of different elements increase with the same slope. Beyond $N=50$, the radii of isotopes near to the proton closedshell such as zinc $(Z=30)$ increase with the same magnitude as the open proton shell isotopes krypton $(Z=36)$ and rubidium $(Z=37)$. These trends are almost identical in the tin region below and beyond the neutron number $N=82$ (see Figure 4iii ).

The rapid increase of the nuclear charge radii observed beyond the neutron number $N=28$ is in contrast with the patterns seen in isotopes close to stability. For neutronrich nuclei in the calcium region, the discontinuities seen in other observables such as $S_{2 n}$ [114] and $E\left(2^{+}\right)$[115] values at neutron number $N=32$, do not appear to be evident in the nuclear charge radii trends. A compilation of different properties measured in the calcium region is shown in Figure 5. The signatures of closed shells at $N=20$ and $N=28$ appears across all observables. For the nuclear charge radii (Figure 5i ) the signatures at $N=20$ are present but less pronounced than for $N=28$. At $N=32$ and $N=34$ the clear agreement for the signs of shell closures among the different observables breaks down, and distinct regular patterns appear for different observables.

Only very recently systematic measurements have been achieved for the nuclear charge radii in the vicinity of calcium and tin isotopes beyond $N=28$ and $N=82$ [101, $124,128]$. The charge radii and electromagnetic moments of ${ }^{58-70} \mathrm{Ni},{ }^{124-134} \mathrm{Sn}$ and ${ }^{112-134} \mathrm{Sb}$ isotopes have been measured by the COLLAPS collaboration at ISOLDECERN [128, 161]. Moreover, results for ${ }^{47-52} \mathrm{~K}(Z=19)$, ${ }^{58-78} \mathrm{Cu}(Z=29),{ }^{104-111} \mathrm{Sn}(Z=50)$ and ${ }^{101-131} \mathrm{In}$ $(Z=49)$ isotopes have been obtained by the CRIS collaboration at ISOLDE-CERN [162-165]. Current efforts to extend these measurements to more exotic calcium, potassium, indium and tin isotopes are underway [163,166-168].

\section{Conclusions}

Despite the drastic difference of interactions between their constituents, the collective properties of strongly correlated many-body systems exhibit surprisingly common features. A common feature is the appearance of shell structures and collective phenomena with seemingly simple trends. From dust particles governed by coulomb interactions, atomic clusters interacting by covalent bonds and interatomic potentials, up to nuclei governed by short-range nuclear forces. The interactions, length scale and dynamics are very different, but these systems present similar signatures of shell structures and collective phenomena. Perhaps the commonalities between these often seemingly disparate many-body systems may further allow mutual advancements in nuclear structure physics, as for example was found in the field of atomic nanoclusters by the successful application of modified nuclear structure models.

The recent developments in many-body theory and the continuous increases in computing power have allowed an unprecedented reductionist insight of the emergence of physical phenomena. This connection between reductionist and emergence viewpoints is grounded uniquely through empirical observations.

The microscopic description of strongly-correlated manybody systems demands different theoretical challenges. In contrast to other quantum systems, the atomic nucleus 


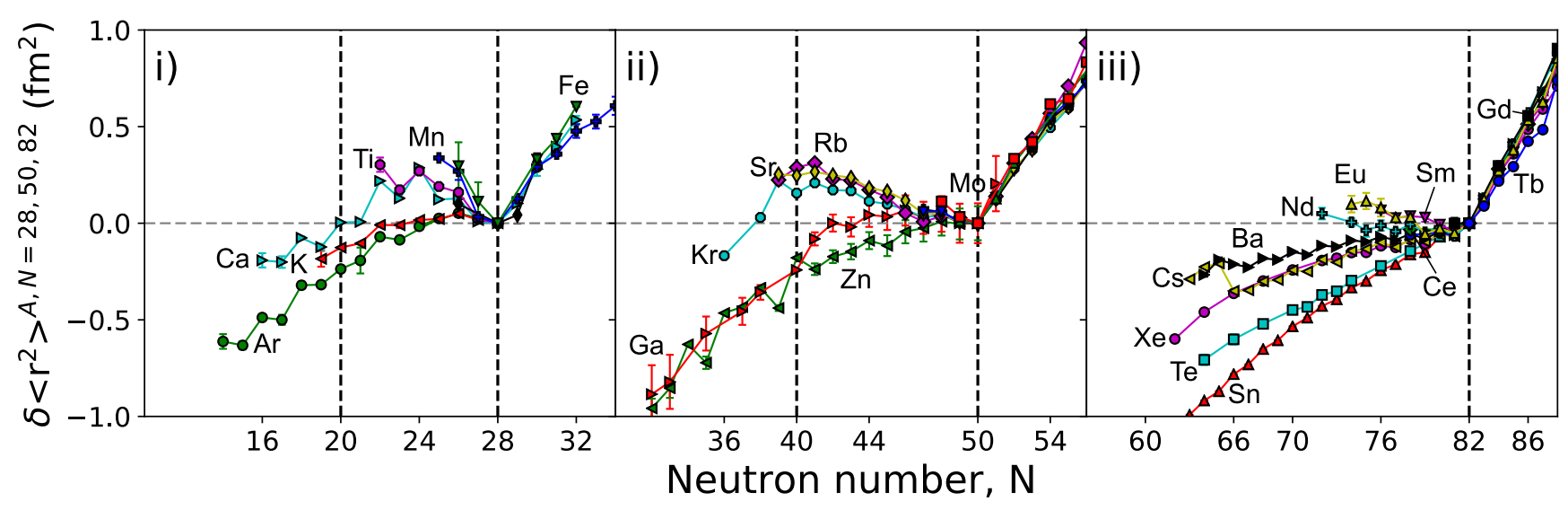

Fig. 4. Changes in the root-mean-square charge radii as a function of the neutron number: i. calcium $(Z=20)$, ii, nickel $(Z=28)$, and iii. tin region $(Z=50)$. Each isotopic chain is shown with respect to the isotope with neutron number at the closed shell. Experimental data were taken from [101,121-130].

is formed by two different constituents (protons and neutrons) that interact mainly by the electromagnetic, strong and weak forces. Recent developments in many-body methods and higher computing power have provided great steps towards the understanding of the microscopic origin of collective phenomena in different regions of the nuclear chart [169-174]. However, a consistent and unified microscopic description of the distinct nuclear phenomena remains as an open problem for nuclear theory. A particular challenge has been the description of nuclear charge radii, where new data in neutron-rich nuclei all exhibit an intriguingly simple increase in charge radii beyond nuclear closed-shells. Moreover, the electromagnetic properties of isotopes around magic numbers of protons and neutrons have been found to exhibit astonishingly simple trends. The microscopic description of these simple patterns, which are predicted by the oldest models of nuclear physics, is a major challenge for modern nuclear theory.

\section{References}

1. Erin G Teich, Greg van Anders, Daphne Klotsa, Julia Dshemuchadse, and Sharon C Glotzer. Clusters of polyhedra in spherical confinement. Proc. Natl. Acad. Sci. U. S. A., 113(6):E669-78, feb 2016.

2. Didier Reinhardt. Phyllotaxis - A new chapter in an old tale about beauty and magic numbers, oct 2005 .

3. Oliver Arp, Dietmar Block, Alexander Piel, and André Melzer. Dust coulomb balls: Three-dimensional plasma crystals. Phys. Rev. Lett., 93(16), oct 2004.

4. I. V. Grigorieva, W. Escoffier, J. Richardson, L. Y. Vinnikov, S. Dubonos, and V. Oboznov. Direct observation of vortex shells and magic numbers in mesoscopic superconducting disks. Phys. Rev. Lett., 96(7), 2006.

5. Junwei Wang, Chrameh Fru Mbah, Thomas Przybilla, Benjamin Apeleo Zubiri, Erdmann Spiecker, Michael Engel, and Nicolas Vogel. Magic number colloidal clusters as minimum free energy structures. Nat. Commun., 9(1), dec 2018
6. Hans E. Suess and Harold C. Urey. Abundances of the Elements. Rev. Mod. Phys., 28(1):53-74, jan 1956.

7. Patrick W. Fowler and David E. Manolopoulos. Magic numbers and stable structures for fullerenes, fullerides and fullerenium ions. Nature, 355(6359):428-430, 1992.

8. Matthias Brack. The physics of simple metal clusters: Self-consistent jellium model and semiclassical approaches. Rev. Mod. Phys., 65(3):677-732, 1993.

9. Walt A. De Heer. The physics of simple metal clusters: Experimental aspects and simple models. Rev. Mod. Phys., 65(3):611-676, 1993.

10. P. W. Anderson. More is different. Science, 177(4047):393-396, 1972.

11. Michael Schmidt and Hod Lipson. Distilling freeform natural laws from experimental data. Science, 324(5923):81-85, 2009.

12. Nigel Goldenfeld and Leo P. Kadanoff. Simple lessons from complexity. Science, 284(5411):87-89, 1999.

13. Igal Talmi. Simple Models of Complex Nuclei. Routledge, 1993.

14. Sophie Gibb, Robin Findlay Hendry, and Tom Lancaster. The Routledge Handbook of Philosophy of Emergence. Routledge, 2018.

15. Piers Coleman. Emergence and Reductionism: an awkward Baconian alliance. arXiv, 1702.06884, feb 2017.

16. J.N. Ridley. Packing efficiency in sunflower heads. Math. Biosci., 58(1):129-139, feb 1982.

17. Helmut Vogel. A better way to construct the sunflower head. Math. Biosci., 44(3-4):179-189, jun 1979.

18. Ting Chen and Sharon C. Glotzer. Simulation studies of a phenomenological model for elongated virus capsid formation. Phys. Rev. E - Stat. Nonlinear, Soft Matter Phys., 75(5), may 2007.

19. W. H. Roos, R. Bruinsma, and G. J.L. Wuite. Physical virology. Nat. Phys., 6(10):733-743, 2010.

20. Bonnie Berger, Peter W. Shor, Lisa Tucker-Kellogg, and Jonathan King. Local rule-based theory of virus shell assembly. Proc. Natl. Acad. Sci. U. S. A., 91(16):77327736, aug 1994.

21. W. Krätschmer, Lowell D. Lamb, K. Fostiropoulos, and Donald R. Huffman. Solid C60: a new form of carbon. Nature, 347(6291):354-358, 1990. 


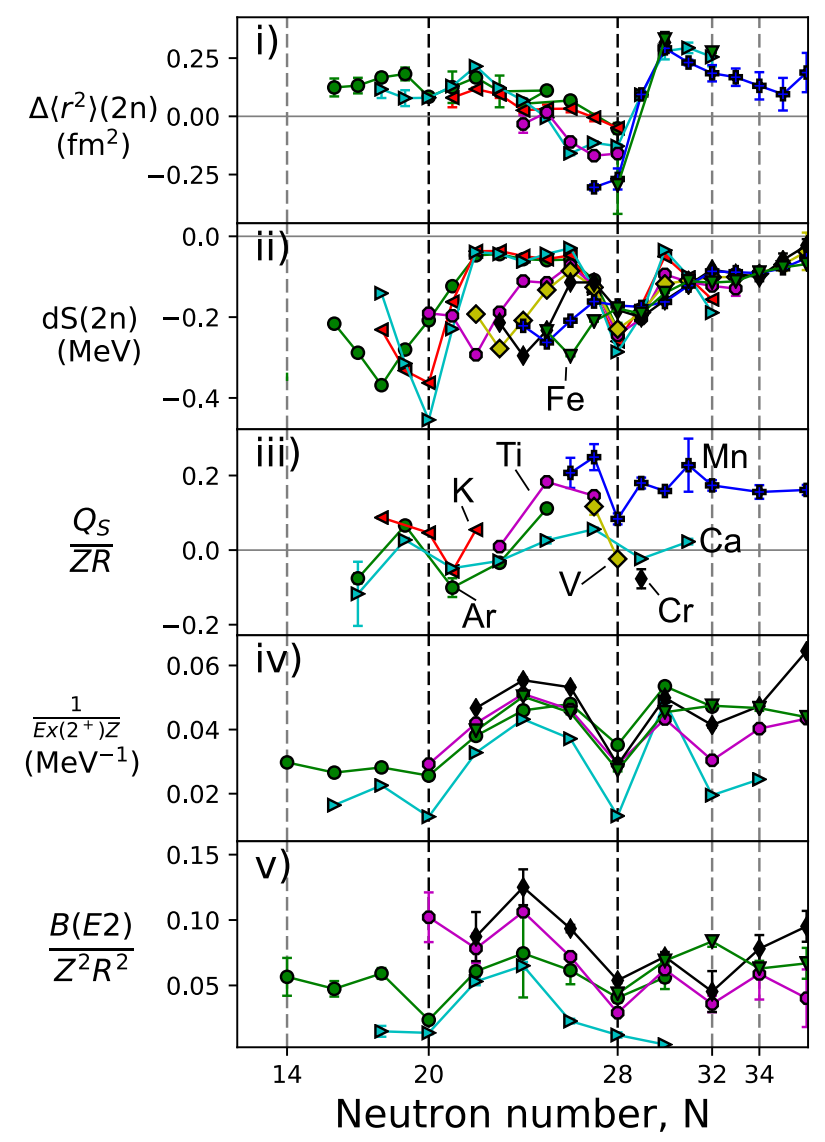

Fig. 5. Experimental nuclear properties in the calcium region: i. root-mean-squared charge radii difference when two neutrons are added, $\left\langle r^{2}\right\rangle(2 \mathrm{n})$; ii. derivative of the two-nucleon separation energy $\mathrm{d} S_{2 n}$; iii. normalized spectroscopic quadrupole moments $Q_{s} / Z R$; iv. scaled inverse of the excitation energy of the first $2^{+}$state, $1 / E_{2+} Z$; and v. normalized transition probability to the first $2^{+}$excited state, $B(E 2) / Z^{2} R^{2}$. Experimental data were taken from $[38,101,113,131-140]$.

22. François Diederich, Roland Ettl, Yves Rubin, Robert L. Whetten, Rainer Beck, Marcos Alvarez, Samir Anz, Dilip Sensharma, Fred Wudl, Kishan C. Khemani, and Andrew Koch. The higher fullerenes: Isolation and characterization of C76, C84, C90, C94, and C70O, an oxide of D5hC70. Science (80-. )., 252(5005):548-551, 1991.

23. Han Myoung Lee, Seung Bum Suh, P. Tarakeshwar, and Kwang S. Kim. Origin of the magic numbers of water clusters with an excess electron. J. Chem. Phys., 122(4), 2005.

24. O. Echt, K. Sattler, and E. Recknagel. Magic Numbers for Sphere Packings: Experimental Verification in Free Xenon Clusters. Phys. Rev. Lett., 47(16):1121-1124, oct 1981.

25. O. Echt, M. C. Cook, and A. W. Castleman. Multiphoton ionization studies of xenon clusters. Chem. Phys. Lett., 135(3):229-235, apr 1987.

26. W. D. Knight, Keith Clemenger, Walt A. de Heer, Winston A. Saunders, M. Y. Chou, and Marvin L. Cohen.
Electronic Shell Structure and Abundances of Sodium Clusters. Phys. Rev. Lett., 52(24):2141-2143, jun 1984.

27. J. Pedersen, S. Bjørnholm, J. Borggreen, K. Hansen, T. P. Martin, and H. D. Rasmussen. Observation of quantum supershells in clusters of sodium atoms. Nature, 353(6346):733-735, 1991.

28. M. L. Homer, J. L. Persson, E. C. Honea, and R. L. Whetten. Ionization energies and stabilities of Nan, $\mathrm{n}<25$ : shell structure from measurements on cold clusters. Zeitschrift für Phys. D Atoms, Mol. Clust., 22(1):441-447, mar 1991.

29. Hellmut Haberland, Thomas Hippler, Jörn Donges, Oleg Kostko, Martin Schmidt, and Bernd Von Issendorff. Melting of sodium clusters: Where do the magic numbers come from? Phys. Rev. Lett., 94(3), jan 2005.

30. NIST ASD Team A. Kramida, Y. Ralchenko, J. Reader. NIST Atomic Spectra Database, 2014.

31. G Audi, M Wang, and A H Wapstra. Chinese Physics C. Chinese Phys. C, 41:30002, 2017.

32. Meng Wang, G Audi, F G Kondev, W J Huang, S Naimi, and Xing $\mathrm{Xu}$. Chinese Physics C. Chinese Phys. C, 41(3):30003, 2017.

33. G. Audi, F. G. Kondev, Meng Wang, W. J. Huang, and S. Naimi. The NUBASE2016 evaluation of nuclear properties. Chinese Phys. C, 41(3), mar 2017.

34. L. W. Nordheim. $\beta$-Decay and the nuclear shell model, 1950.

35. M. Ismail, W. M. Seif, and A. Abdurrahman. Relative stability and magic numbers of nuclei deduced from behavior of cluster emission half-lives. Phys. Rev. C, 94(2), aug 2016.

36. I. Angeli and K.P. Marinova. Table of experimental nuclear ground state charge radii: An update. At. Data Nucl. Data Tables, 99(1):69-95, jan 2013.

37. B. Pritychenko, M. Birch, B. Singh, and M. Horoi. Tables of E2 transition probabilities from the first 2+ states in even-even nuclei. At. Data Nucl. Data Tables, 107:1-139, jan 2016.

38. N. J. Stone. Table of nuclear electric quadrupole moments. At. Data Nucl. Data Tables, 111-112:1-28, sep 2016.

39. N. Grevesse and A.J. Sauval. Standard Solar Composition. Space Sci. Rev., 85(1/2):161-174, 1998.

40. H. Hurwitz and H. A. Bethe. Neutron capture cross sections and level density, 1951.

41. Duncan E.K. Sutherland and Martin J. Stillman. The "magic numbers" of metallothionein, may 2011.

42. D. Marenduzzo, C. Micheletti, and E. Orlandini. Biopolymer organization upon confinement. J. Phys. Condens. Matter, 22(28), jul 2010.

43. R. John Ellis. Macromolecular crowding: Obvious but underappreciated, oct 2001.

44. A M Turing. The Chemical Basis of Morphogenesis. Technical Report 641, 1952.

45. Hajar Vakili, Pavel Kroupa, and Sohrab Rahvar. Type I Shell Galaxies as a Test of Gravity Models. Astrophys. J., 848(1):55, oct 2017.

46. Douglas B. Cines, Tatiana Lebedeva, Chandrasekaran Nagaswami, Vincent Hayes, Walter Massefski, Rustem I. Litvinov, Lubica Rauova, Thomas J. Lowery, and John W. Weisel. Clot contraction: Compression of erythrocytes into tightly packed polyhedra and redistribution of platelets and fibrin. Blood, 123(10):1596-1603, mar 2014 . 
47. Takashi Hayashi and Richard W. Carthew. Surface mechanics mediate pattern formation in the developing retina. Nature, 431(7009):647-652, oct 2004.

48. J. A.S. Kelso and G. Schöner. Self-organization of coordinative movement patterns. Hum. Mov. Sci., 7(1):27-46, 1988.

49. G. Schöner and J. A.S. Kelso. Dynamic pattern generation in behavioral and neural systems. Science (80-. )., 239(4847):1513-1520, 1988.

50. G. J. Mitchison. Phyllotaxis and the fibonacci series. Science (80-. )., 196(4287):270-275, 1977.

51. Didier Reinhardt, Eva Rachele Pesce, Pia Stieger, Therese Mandel, Kurt Baltensperger, Malcolm Bennett, Jan Traas, Jií Friml, and Cris Kuhlemeier. Regulation of phyllotaxis by polar auxin transport. Nature, 426(6964):255-260, nov 2003.

52. Reza Farhadifar, Jens Christian Röper, Benoit Aigouy, Suzanne Eaton, and Frank Jülicher. The Influence of Cell Mechanics, Cell-Cell Interactions, and Proliferation on Epithelial Packing. Curr. Biol., 17(24):2095-2104, dec 2007.

53. Hiroaki Ohfuji and David Rickard. Experimental syntheses of framboids - A review. Earth-Science Rev., 71(34):147-170, aug 2005 .

54. Hiroaki Ohfuji and Junji Akai. Icosahedral domain structure of framboidal pyrite. Am. Mineral., 87(1):176-180, 2002.

55. S. Elizabeth Norred, Patrick M. Caveney, Gaurav Chauhan, Lauren K. Collier, C. Patrick Collier, Steven M. Abel, and Michael L. Simpson. Macromolecular Crowding Induces Spatial Correlations That Control Gene Expression Bursting Patterns. ACS Synth. Biol., 7(5):12511258, may 2018.

56. R. John Ellis and Allen P. Minton. Join the crowd, sep 2003.

57. Cristian Micheletti, Davide Marenduzzo, and Enzo Orlandini. Polymers with spatial or topological constraints: Theoretical and computational results, jul 2011.

58. Ralph A. Alpher and Robert C. Herman. Theory of the origin and relative abundance distribution of the elements. Rev. Mod. Phys., 22(2):153-212, 1950.

59. Edward Anders and Nicolas Grevesse. Abundances of the elements: Meteoritic and solar. Geochim. Cosmochim. Acta, 53(1):197-214, 1989.

60. B. E. J. Pagel. Nucleosynthesis and chemical evolution of galaxies. Cambridge University Press, 2009.

61. R. McNeill. Alexander. Optima for animals. Princeton University Press, 1996.

62. J. M. Cherrett, A. D. Bradshaw, F. B. Goldsmith, P. J. Grubb, and J. R. Krebs. Ecological concepts: the contribution of ecology to an understanding of the natural world. Blackwell Scientific, for British Ecological Society; Symposium, 29, 1989.

63. J Maynard Smith. Evolution and the Theory of Games: In situations characterized by conflict of interest, the best strategy to adopt depends on what others are doing. Am. Sci., 64(1):41-45, 1976.

64. G. A. Parker and J. Maynard Smith. Optimality theory in evolutionary biology, 1990.

65. Michael A. Boles, Michael Engel, and Dmitri V. Talapin. Self-assembly of colloidal nanocrystals: From intricate structures to functional materials, sep 2016.
66. R. Rutgers. Packing of spheres. Nature, 193(4814):465466, 1962 .

67. Ignacio Castillo, Frank J. Kampas, and János D. Pintér. Solving circle packing problems by global optimization: Numerical results and industrial applications. Eur. J. Oper. Res., 191(3):786-802, dec 2008.

68. Maria Goeppert Mayer. On Closed Shells in Nuclei. II. Phys. Rev., 75(12):1969-1970, jun 1949.

69. Otto Haxel, J. Hans D. Jensen, and Hans E. Suess. On the "magic numbers" in nuclear structure, 1949.

70. M. Bonitz, C. Henning, and D. Block. Complex plasmas: A laboratory for strong correlations. Reports Prog. Phys., 73(6), 2010.

71. V. E. Fortov, A. V. Ivlev, S. A. Khrapak, A. G. Khrapak, and G. E. Morfill. Complex (dusty) plasmas: Current status, open issues, perspectives, dec 2005.

72. O Arp, D Block, M Bonitz, H Fehske, V Golubnychiy, S Kosse, P Ludwig, A Melzer, and A Piel. 3D Coulomb balls: experiment and simulation. J. Phys. Conf. Ser., 11:234-247, jan 2005.

73. O. Arp, D. Block, M. Klindworth, and A. Piel. Confinement of Coulomb balls. Phys. Plasmas, 12(12):1-9, 2005.

74. P. Ludwig, S. Kosse, and M. Bonitz. Structure of spherical three-dimensional Coulomb crystals. Phys. Rev. E Stat. Nonlinear, Soft Matter Phys., 71(4), apr 2005.

75. Wen Tau Juan, Zen Hong Huang, Ju Wang Hsu, Yin Ju Lai, and I. Lin. Observation of dust Coulomb clusters in a plasma trap. Phys. Rev. E, 58(6):R6947-R6950, 1998.

76. A. D. Dinsmore, Ming F. Hsu, M. G. Nikolaides, Manuel Marquez, A. R. Bausch, and D. A. Weitz. Colloidosomes: Selectively permeable capsules composed of colloidal particles. Science (80-. )., 298(5595):1006-1009, nov 2002.

77. Bart De Nijs, Simone Dussi, Frank Smallenburg, Johannes D. Meeldijk, Dirk J. Groenendijk, Laura Filion, Arnout Imhof, Alfons Van Blaaderen, and Marjolein Dijkstra. Entropy-driven formation of large icosahedral colloidal clusters by spherical confinement. Nat. Mater., 14(1):56-60, jan 2015.

78. Vinothan N. Manoharan, Mark T. Elsesser, and David J. Pine. Dense packing and symmetry in small clusters of microspheres. Science (80-. )., 301(5632):483-487, jul 2003.

79. Noel A. Clark, Alan J. Hurd, and Bruce J. Ackerson. Single colloidal crystals, 1979.

80. Orlin D. Velev, Abraham M. Lenhoff, and Eric W. Kaler. A class of microstructured particles through colloidal crystallization. Science (80-. )., 287(5461):2240-2243, mar 2000 .

81. T.P. Martin. Shells of atoms. Phys. Rep., 273(4):199-241, aug 1996.

82. Matthias Brack. Metal Clusters and Magic Numbers. 277(6):50-55, 1997.

83. Marvin L. Cohen and Walter D. Knight. The Physics of Metal Clusters. Phys. Today, 43(12):42-50, dec 1990.

84. H. Nishioka, Klavs Hansen, and B. R. Mottelson. Supershells in metal clusters. Phys. Rev. B, 42(15):9377-9386, 1990.

85. I. Katakuse, T. Ichihara, Y. Fujita, T. Matsuo, T. Sakurai, and H. Matsuda. Mass distributions of copper, silver and gold clusters and electronic shell structure. Int. J. Mass Spectrom. Ion Process., 67(2):229-236, nov 1985.

86. W. Ekardt. Work function of small metal particles: Selfconsistent spherical jellium-background model. Phys. Rev. B, 29(4):1558-1564, feb 1984. 
87. Mark A. Stoyer. Island ahoy! Nature, 442(7105):876-877, aug 2006.

88. Walt A. De Heer. The physics of simple metal clusters: Experimental aspects and simple models. Rev. Mod. Phys., 65(3):611-676, 1993.

89. S.G. Nilsson. Binding states of individual nucleons in strongly deformed nuclei. Kong.Dan.Vid.Sel.Mat.Fys.Med., 29N16:1-69, 1955.

90. Keith Clemenger. Ellipsoidal shell structure in freeelectron metal clusters. Phys. Rev. B, 32(2):1359-1362, 1985.

91. B. L. Berman and S. C. Fultz. Measurements of the giant dipole resonance with monoenergetic photons. Rev. Mod. Phys., 47(3):713-761, jul 1975.

92. W. Ekardt. Collective multipole excitations in small metal particles: Critical angular momentum for the existence of collective surface modes. Phys. Rev. B, 32(4):1961-1970, aug 1985.

93. Søren Raza, Shima Kadkhodazadeh, Thomas Christensen, Marcel Di Vece, Martijn Wubs, N. Asger Mortensen, and Nicolas Stenger. Multipole plasmons and their disappearance in few-nanometre silver nanoparticles. Nat. Commun., 6(1):8788, dec 2015.

94. Eric C. Honea, Margie L. Homer, John L. Persson, and Robert L. Whetten. Generation and photoionization of cold Nan clusters; n to 200. Chem. Phys. Lett., 171(3):147-154, aug 1990.

95. M. P. Iñiguez, J. A. Alonso, and L. C. Balbas. Magic numbers of sodium clusters. Solid State Commun., 57(1):8588, 1986.

96. G. Wrigge, M. Astruc Hoffmann, and B. V. Issendorff. Photoelectron spectroscopy of sodium clusters: Direct observation of the electronic shell structure. Phys. Rev. A At. Mol. Opt. Phys., 65(6):5, 2002.

97. M Schmidt, J Donges, Th Hippler, and H Haberland. Influence of Energy and Entropy on the Melting of Sodium Clusters. 2003.

98. Hans-Werner Hammer, Andreas Nogga, and Achim Schwenk. Colloquium: Three-body forces: From cold atoms to nuclei. Rev. Mod. Phys., 85:197-217, Jan 2013.

99. Kris Heyde and John L. Wood. Shape coexistence in atomic nuclei. Rev. Mod. Phys., 83:1467-1521, Nov 2011.

100. Z.-T. Lu, P. Mueller, G. W. F. Drake, W. Nörtershäuser, Steven C. Pieper, and Z.-C. Yan. Colloquium: Laser probing of neutron-rich nuclei in light atoms. Rev. Mod. Phys., 85:1383-1400, Oct 2013.

101. R. F. Garcia Ruiz, M. L. Bissell, K. Blaum, A. Ekström, N. Frömmgen, G. Hagen, M. Hammen, K. Hebeler, J. D. Holt, G. R. Jansen, M. Kowalska, K. Kreim, W. Nazarewicz, R. Neugart, G. Neyens, W. Nörtershäuser, T. Papenbrock, J. Papuga, A. Schwenk, J. Simonis, K.A. Wendt, and D. T. Yordanov. Unexpectedly large charge radii of neutronrich calcium isotopes. Nature Physics, 12(6):594-598, jun 2016 .

102. Martin Freer, Hisashi Horiuchi, Yoshiko Kanada-En'yo, Dean Lee, and Ulf-G. Meißner. Microscopic clustering in light nuclei. Rev. Mod. Phys., 90:035004, Aug 2018.

103. J. A. S. Smith. Nuclear quadrupole interactions in solids. Chem. Soc. Rev., 15:225-260, 1986.

104. R. F. Casten, N. V. Zamfir, and D. S. Brenner. Universal anharmonic vibrator description of nuclei and critical nuclear phase transitions. Phys. Rev. Lett., 71:227-230, Jul 1993.
105. O. Sorlin and M.-G. Porquet. Nuclear magic numbers: New features far from stability. Progress in Particle and Nuclear Physics, 61(2):602 - 673, 2008.

106. I Angeli and K P Marinova. Correlations of nuclear charge radii with other nuclear observables. Journal of Physics G: Nuclear and Particle Physics, 42(5):055108, mar 2015.

107. I. Bentley, Y. Colón Rodríguez, S. Cunningham, and A. Aprahamian. Shell structure from nuclear observables. Phys. Rev. C, 93:044337, Apr 2016.

108. R. F. Casten. Symmetries and regularities in nuclei: Order out of seeming chaos. AIP Conference Proceedings, 2150:020001, 2019.

109. Maria Goeppert Mayer. Nuclear configurations in the spin-orbit coupling model. i. empirical evidence. Phys. Rev., 78:16-21, Apr 1950.

110. Maria Goeppert Mayer. Nuclear configurations in the spin-orbit coupling model. ii. theoretical considerations. Phys. Rev., 78:22-23, Apr 1950.

111. A. Bohr and B.R. Mottelson. Nuclear Structure, volume I II. Benjamin,New York, 1975.

112. R. B. Cakirli, R. F. Casten, and K. Blaum. Correlations of experimental isotope shifts with spectroscopic and mass observables. Phys. Rev. C, 82:061306, Dec 2010.

113. R. F. Garcia Ruiz, M. L. Bissell, K. Blaum, N. Frömmgen, M. Hammen, J. D. Holt, M. Kowalska, K. Kreim, J. Menéndez, R. Neugart, G. Neyens, W. Nörtershäuser, F. Nowacki, J. Papuga, A. Poves, A. Schwenk, J. Simonis, and D. T. Yordanov. Ground-state electromagnetic moments of calcium isotopes. Phys. Rev. C, 91:041304, Apr 2015.

114. F. Wienholtz, D. Beck, K. Blaum, Ch. Borgmann, M. Breitenfeldt, R. B. Cakirli, S. George, F. Herfurth, J. D. Holt, M. Kowalska, S. Kreim, D. Lunney, V. Manea, J. Menéndez, D. Neidherr, M. Rosenbusch, L. Schweikhard, A. Schwenk, J. Simonis, J. Stanja, R. N. Wolf, and K. Zuber. Masses of exotic calcium isotopes pin down nuclear forces. Nature, 498(7454):346-349, jun 2013.

115. D. Steppenbeck et al. Evidence for a new nuclear magic number from the level structure of 54Ca. Nature, 502:207, 2013.

116. J. Eberz, U. Dinger, G. Huber, H. Lochmann, R. Menges, R. Neugart, R. Kirchner, O. Klepper, T. Khl, D. Marx, G. Ulm, and K. Wendt. Spins, moments and mean square charge radii of 104127in determined by laser spectroscopy. Nuclear Physics A, 464(1):9 - 28, 1987.

117. F. Le Blanc, L. Cabaret, E. Cottereau, J.E. Crawford, S. Essabaa, J. Genevey, R. Horn, G. Huber, J. Lassen, J.K.P. Lee, G. Le Scornet, J. Obert, J. Oms, A. Ouchrif, J. Pinard, B. Roussie're, J. Sauvage, and D. Verney. Charge radii and nuclear moments around132sn. Nuclear Physics A, 734:437 - 440, 2004.

118. D. T. Yordanov, D. L. Balabanski, J. Bieroń, M. L. Bissell, K. Blaum, I. Budinčević, S. Fritzsche, N. Frömmgen, G. Georgiev, Ch. Geppert, M. Hammen, M. Kowalska, K. Kreim, A. Krieger, R. Neugart, W. Nörtershäuser, J. Papuga, and S. Schmidt. Spins, electromagnetic moments, and isomers of ${ }^{107-129} \mathrm{Cd}$. Phys. Rev. Lett., 110:192501, May 2013.

119. R. F. Garcia Ruiz, A. R. Vernon, C. L. Binnersley, B. K. Sahoo, M. Bissell, J. Billowes, T. E. Cocolios, W. Gins, R. P. de Groote, K. T. Flanagan, A. Koszorus, K. M. Lynch, G. Neyens, C. M. Ricketts, K. D. A. Wendt, S. G. 
Wilkins, and X. F. Yang. High-precision multiphoton ionization of accelerated laser-ablated species. Phys. Rev. $X, 8: 041005$, Oct 2018.

120. M. Lindroos, M. Booth, D. Doran, Y. Koh, I. Oliveira, J. Rikovska, P. Richards, N. J. Stone, M. Veskovic, D. Zákoucký, and B. Fogelberg. Magnetic dipole moment of $127 \mathrm{Sb}$ and $129 \mathrm{Sb}$ by nuclear magnetic resonance on oriented nuclei. Phys. Rev. C - Nucl. Phys., 53(1):124-126, 1996.

121. K. Kreim, M.L. Bissell, J. Papuga, K. Blaum, M. De Rydt, R.F. Garcia Ruiz, S. Goriely, H. Heylen, M. Kowalska, R. Neugart, G. Neyens, W. Nrtershuser, M.M. Rajabali, R. Snchez Alarcn, H.H. Stroke, and D.T. Yordanov. Nuclear charge radii of potassium isotopes beyond $n=28$. Physics Letters B, 731:97 - 102, 2014.

122. M. L. Bissell, J. Papuga, H. Naïdja, K. Kreim, K. Blaum, M. De Rydt, R. F. Garcia Ruiz, H. Heylen, M. Kowalska, R. Neugart, G. Neyens, W. Nörtershäuser, F. Nowacki, M. M. Rajabali, R. Sanchez, K. Sieja, and D. T. Yordanov. Proton-neutron pairing correlations in the selfconjugate nucleus ${ }^{38} \mathrm{~K}$ probed via a direct measurement of the isomer shift. Phys. Rev. Lett., 113:052502, Jul 2014.

123. X. F. Yang, C. Wraith, L. Xie, C. Babcock, J. Billowes, M. L. Bissell, K. Blaum, B. Cheal, K. T. Flanagan, R. F. Garcia Ruiz, W. Gins, C. Gorges, L. K. Grob, H. Heylen, S. Kaufmann, M. Kowalska, J. Kraemer, S. MalbrunotEttenauer, R. Neugart, G. Neyens, W. Nörtershäuser, J. Papuga, R. Sánchez, and D. T. Yordanov. Isomer shift and magnetic moment of the long-lived $1 / 2^{+}$isomer in ${ }_{30}^{79} \mathrm{Zn}_{49}$ : Signature of shape coexistence near ${ }^{78} \mathrm{Ni}$. Phys. Rev. Lett., 116:182502, May 2016.

124. H. Heylen, C. Babcock, R. Beerwerth, J. Billowes, M. L. Bissell, K. Blaum, J. Bonnard, P. Campbell, B. Cheal, T. Day Goodacre, D. Fedorov, S. Fritzsche, R. F. Garcia Ruiz, W. Geithner, Ch. Geppert, W. Gins, L. K. Grob, M. Kowalska, K. Kreim, S. M. Lenzi, I. D. Moore, B. Maass, S. Malbrunot-Ettenauer, B. Marsh, R. Neugart, G. Neyens, W. Nörtershäuser, T. Otsuka, J. Papuga, R. Rossel, S. Rothe, R. Sánchez, Y. Tsunoda, C. Wraith, L. Xie, X. F. Yang, and D. T. Yordanov. Changes in nuclear structure along the $\mathrm{mn}$ isotopic chain studied via charge radii. Phys. Rev. C, 94:054321, Nov 2016.

125. K. Minamisono, D. M. Rossi, R. Beerwerth, S. Fritzsche, D. Garand, A. Klose, Y. Liu, B. Maaß, P. F. Mantica, A. J. Miller, P. Müller, W. Nazarewicz, W. Nörtershäuser, E. Olsen, M. R. Pearson, P.-G. Reinhard, E. E. Saperstein, C. Sumithrarachchi, and S. V. Tolokonnikov. Charge radii of neutron deficient ${ }^{52,53} \mathrm{Fe}$ produced by projectile fragmentation. Phys. Rev. Lett., 117:252501, Dec 2016.

126. G. J. Farooq-Smith, A. R. Vernon, J. Billowes, C. L. Binnersley, M. L. Bissell, T. E. Cocolios, T. Day Goodacre, R. P. de Groote, K. T. Flanagan, S. Franchoo, R. F. Garcia Ruiz, W. Gins, K. M. Lynch, B. A. Marsh, G. Neyens, S. Rothe, H. H. Stroke, S. G. Wilkins, and X. F. Yang. Probing the ${ }_{31} \mathrm{Ga}$ ground-state properties in the region near $z=28$ with high-resolution laser spectroscopy. Phys. Rev. C, 96:044324, Oct 2017.

127. M. Hammen, W. Nörtershäuser, D. L. Balabanski, M. L. Bissell, K. Blaum, I. Budinčević, B. Cheal, K. T. Flanagan, N. Frömmgen, G. Georgiev, Ch. Geppert, M. Kowalska, K. Kreim, A. Krieger, W. Nazarewicz, R. Neugart, G. Neyens, J. Papuga, P.-G. Reinhard, M. M. Rajabali,
S. Schmidt, and D. T. Yordanov. From Calcium to Cadmium: Testing the Pairing Functional through Charge Radii Measurements of Cd100-130. Phys. Rev. Lett., 121(10):102501, 2018.

128. C. Gorges, L. V. Rodríguez, D. L. Balabanski, M. L. Bissell, K. Blaum, B. Cheal, R. F. Garcia Ruiz, G. Georgiev, W. Gins, H. Heylen, A. Kanellakopoulos, S. Kaufmann, M. Kowalska, V. Lagaki, S. Lechner, B. Maaß, S. Malbrunot-Ettenauer, W. Nazarewicz, R. Neugart, G. Neyens, W. Nörtershäuser, P.-G. Reinhard, S. Sailer, R. Sánchez, S. Schmidt, L. Wehner, C. Wraith, L. Xie, Z. Y. Xu, X. F. Yang, and D. T. Yordanov. Laser spectroscopy of neutron-rich tin isotopes: A discontinuity in charge radii across the $n=82$ shell closure. Phys. Rev. Lett., 122:192502, May 2019.

129. A. J. Miller, K. Minamisono, A. Klose, D. Garand, C. Kujawa, J. D. Lantis, Y. Liu, B. Maa, P. F. Mantica, W. Nazarewicz, W. Nrtershuser, S. V. Pineda, P. G. Reinhard, D. M. Rossi, F. Sommer, C. Sumithrarachchi, A. Teigelhfer, and J. Watkins. Proton superfluidity and charge radii in proton-rich calcium isotopes. Nature Physics, 15(5), 22019.

130. L. Xie, X.F. Yang, C. Wraith, C. Babcock, J. Biero, J. Billowes, M.L. Bissell, K. Blaum, B. Cheal, L. Filippin, K.T. Flanagan, R.F. Garcia Ruiz, W. Gins, G. Gaigalas, M. Godefroid, C. Gorges, L.K. Grob, H. Heylen, P. Jnsson, S. Kaufmann, M. Kowalska, J. Krmer, S. MalbrunotEttenauer, R. Neugart, G. Neyens, W. Nrtershuser, T. Otsuka, J. Papuga, R. Snchez, Y. Tsunoda, and D.T. Yordanov. Nuclear charge radii of ${ }^{62-80} \mathrm{Zn}$ and their dependence on cross-shell proton excitations. Physics Letters B, 797:134805, 2019 .

131. National Nuclear Data Center. NNDC. https://www.nndc.bnl.gov/, 2019.

132. C. Babcock, H. Heylen, M.L. Bissell, K. Blaum, P. Campbell, B. Cheal, D. Fedorov, R.F. Garcia Ruiz, W. Geithner, W. Gins, T. Day Goodacre, L.K. Grob, M. Kowalska, S.M. Lenzi, B. Maass, S. Malbrunot-Ettenauer, B. Marsh, R. Neugart, G. Neyens, W. Nrtershuser, T. Otsuka, R. Rossel, S. Rothe, R. Snchez, Y. Tsunoda, C. Wraith, L. Xie, and X.F. Yang. Quadrupole moments of odda 5363mn: Onset of collectivity towards $\mathrm{n}=40$. Physics Letters B, 760:387 - 392, 2016.

133. X. F. Yang, Y. Tsunoda, C. Babcock, J. Billowes, M. L. Bissell, K. Blaum, B. Cheal, K. T. Flanagan, R. F. Garcia Ruiz, W. Gins, C. Gorges, L. K. Grob, H. Heylen, S. Kaufmann, M. Kowalska, J. Krämer, S. MalbrunotEttenauer, R. Neugart, G. Neyens, W. Nörtershäuser, T. Otsuka, J. Papuga, R. Sánchez, C. Wraith, L. Xie, and D. T. Yordanov. Investigating the large deformation of the $5 / 2^{+}$isomeric state in ${ }^{73} \mathrm{Zn}$ : An indicator for triaxiality. Phys. Rev. C, 97:044324, Apr 2018.

134. M. Mougeot, D. Atanasov, K. Blaum, K. Chrysalidis, T. Day Goodacre, D. Fedorov, V. Fedosseev, S. George, F. Herfurth, J. D. Holt, D. Lunney, V. Manea, B. Marsh, D. Neidherr, M. Rosenbusch, S. Rothe, L. Schweikhard, A. Schwenk, C. Seiffert, J. Simonis, S. R. Stroberg, A. Welker, F. Wienholtz, R. N. Wolf, and K. Zuber. Precision Mass Measurements of Cr 58-63: Nuclear Collectivity Towards the $\mathrm{N}=40$ Island of Inversion. Phys. Rev. Lett., 120(23), jun 2018.

135. S. Michimasa, M. Kobayashi, Y. Kiyokawa, S. Ota, D. S. Ahn, H. Baba, G. P.A. Berg, M. Dozono, 
N. Fukuda, T. Furuno, E. Ideguchi, N. Inabe, T. Kawabata, S. Kawase, K. Kisamori, K. Kobayashi, T. Kubo, Y. Kubota, C. S. Lee, M. Matsushita, H. Miya, A. Mizukami, H. Nagakura, D. Nishimura, H. Oikawa, H. Sakai, Y. Shimizu, A. Stolz, H. Suzuki, M. Takaki, H. Takeda, S. Takeuchi, H. Tokieda, T. Uesaka, K. Yako, Y. Yamaguchi, Y. Yanagisawa, R. Yokoyama, K. Yoshida, and S. Shimoura. Magic Nature of Neutrons in Ca 54: First Mass Measurements of Ca 55-57. Phys. Rev. Lett., 121(2), jul 2018.

136. M. P. Reiter, S. Ayet San Andrés, E. Dunling, B. Kootte, E. Leistenschneider, C. Andreoiu, C. Babcock, B. R. Barquest, J. Bollig, T. Brunner, I. Dillmann, A. Finlay, G. Gwinner, L. Graham, J. D. Holt, C. Hornung, C. Jesch, R. Klawitter, Y. Lan, D. Lascar, J. E. McKay, S. F. Paul, R. Steinbrügge, R. Thompson, J. L. Tracy, M. E. Wieser, C. Will, T. Dickel, W. R. Plaß, C. Scheidenberger, A. A. Kwiatkowski, and J. Dilling. Quenching of the $\mathrm{N}=32$ neutron shell closure studied via precision mass measurements of neutron-rich vanadium isotopes. Phys. Rev. C, 98(2), aug 2018.

137. A. Klose, K. Minamisono, A. J. Miller, B. A. Brown, D. Garand, J. D. Holt, J. D. Lantis, Y. Liu, B. Maaß, W. Nörtershäuser, S. V. Pineda, D. M. Rossi, A. Schwenk, F. Sommer, C. Sumithrarachchi, A. Teigelhöfer, and J. Watkins. Ground-state electromagnetic moments of Ca 37. Phys. Rev. C, 99(6), jun 2019.

138. H. N. Liu, A. Obertelli, P. Doornenbal, C. A Bertulani, G. Hagen, J. D. Holt, G. R. Jansen, T. D. Morris, A. Schwenk, R. Stroberg, N. Achouri, H. Baba, F. Browne, D. Calvet, F. Château, S. Chen, N. Chiga, A. Corsi, M. L. Cortés, A. Delbart, J. M. Gheller, A. Giganon, A. Gillibert, C. Hilaire, T. Isobe, T. Kobayashi, Y. Kubota, V. Lapoux, T. Motobayashi, I. Murray, H. Otsu, V. Panin, N. Paul, W. Rodriguez, H. Sakurai, M. Sasano, D. Steppenbeck, L. Stuhl, Y. L. Sun, Y. Togano, T. Uesaka, K. Wimmer, K. Yoneda, O. Aktas, T. Aumann, L. X. Chung, F. Flavigny, S. Franchoo, I. Gašparić, R. B. Gerst, J. Gibelin, K. I. Hahn, D. Kim, T. Koiwai, Y. Kondo, P. Koseoglou, J. Lee, C. Lehr, B. D. Linh, T. Lokotko, M. Maccormick, K. Moschner, T. Nakamura, S. Y. Park, D. Rossi, E. Sahin, D. Sohler, P. A. Söderström, S. Takeuchi, H. Törnqvist, V. Vaquero, V. Wagner, S. Wang, V. Werner, X. Xu, H. Yamada, D. Yan, Z. Yang, M. Yasuda, and L. Zanetti. How Robust is the N=34 Subshell Closure? First Spectroscopy of Ar 52. Phys. Rev. Lett., 122(7), feb 2019.

139. X. Xu, M. Wang, K. Blaum, J. D. Holt, Yu A. Litvinov, A. Schwenk, J. Simonis, S. R. Stroberg, Y. H. Zhang, H. S. Xu, P. Shuai, X. L. Tu, X. H. Zhou, F. R. Xu, G. Audi, R. J. Chen, X. C. Chen, C. Y. Fu, Z. Ge, W. J. Huang, S. Litvinov, D. W. Liu, Y. H. Lam, X. W. Ma, R. S. Mao, A. Ozawa, B. H. Sun, Y. Sun, T. Uesaka, G. Q. Xiao, Y. M. Xing, T. Yamaguchi, Y. Yamaguchi, X. L. Yan, Q. Zeng, H. W. Zhao, T. C. Zhao, W. Zhang, and W. L. Zhan. Masses of neutron-rich Sc 52-54 and Ti 54,56 nuclides: The $\mathrm{N}=32$ subshell closure in scandium. Phys. Rev. C, 99(6), jun 2019.

140. E. Leistenschneider, M. P. Reiter, S. Ayet San Andrés, B. Kootte, J. D. Holt, P. Navrátil, C. Babcock, C. Barbieri, B. R. Barquest, J. Bergmann, J. Bollig, T. Brunner, E. Dunling, A. Finlay, H. Geissel, L. Graham, F. Greiner,
H. Hergert, C. Hornung, C. Jesch, R. Klawitter, Y. Lan, D. Lascar, K. G. Leach, W. Lippert, J. E. McKay, S. F. Paul, A. Schwenk, D. Short, J. Simonis, V. Somà, R. Steinbrügge, S. R. Stroberg, R. Thompson, M. E. Wieser, C. Will, M. Yavor, C. Andreoiu, T. Dickel, I. Dillmann, G. Gwinner, W. R. Plaß, C. Scheidenberger, A. A. Kwiatkowski, and J. Dilling. Dawning of the $\mathrm{N}=32$ Shell Closure Seen through Precision Mass Measurements of Neutron-Rich Titanium Isotopes. Phys. Rev. Lett., 120(6), feb 2018.

141. T. Motobayashi, Y. Ikeda, K. Ieki, M. Inoue, N. Iwasa, T. Kikuchi, M. Kurokawa, S. Moriya, S. Ogawa, H. Murakami, S. Shimoura, Y. Yanagisawa, T. Nakamura, Y. Watanabe, M. Ishihara, T. Teranishi, H. Okuno, and R.F. Casten. Large deformation of the very neutron-rich nucleus $32 \mathrm{mg}$ from intermediate-energy coulomb excitation. Physics Letters B, 346(1):9 - 14, 1995.

142. Tomoaki Togashi, Yusuke Tsunoda, Takaharu Otsuka, and Noritaka Shimizu. Quantum phase transition in the shape of zr isotopes. Phys. Rev. Lett., 117:172502, Oct 2016.

143. Mihai Horoi and Koblar A. Jackson. Signature of shape transition and shape coexistence in mesoscopic systems. Chemical Physics Letters, 427(1):147 - 152, 2006.

144. Gerda Neyens. Nuclear magnetic and quadrupole moments for nuclear structure research on exotic nuclei. Reports on Progress in Physics, 66(4):633-689, mar 2003.

145. J. Wood. Simple structure in complex nuclei. Physics, 6:52, 2013.

146. Ben Mottelson. Elementary modes of excitation in the nucleus. Rev. Mod. Phys., 48:375-383, Jul 1976.

147. Aage Bohr. Rotational motion in nuclei. Rev. Mod. Phys., 48:365-374, Jul 1976.

148. James Rainwater. Background for the spheroidal nuclear model proposal. Rev. Mod. Phys., 48:385-391, Jul 1976.

149. J. Papuga, M. L. Bissell, K. Kreim, K. Blaum, B. A. Brown, M. De Rydt, R. F. Garcia Ruiz, H. Heylen, M. Kowalska, R. Neugart, G. Neyens, W. Nörtershäuser, T. Otsuka, M. M. Rajabali, R. Sánchez, Y. Utsuno, and D. T. Yordanov. Spins and magnetic moments of ${ }^{49} \mathbf{K}$ and ${ }^{51} \mathbf{K}$ : Establishing the $1 / 2^{+}$and $3 / 2^{+}$level ordering beyond $n=28$. Phys. Rev. Lett., 110:172503, Apr 2013.

150. J. Papuga, M. L. Bissell, K. Kreim, C. Barbieri, K. Blaum, M. De Rydt, T. Duguet, R. F. Garcia Ruiz, H. Heylen, M. Kowalska, R. Neugart, G. Neyens, W. Nörtershäuser, M. M. Rajabali, R. Sánchez, N. Smirnova, V. Somà, and D. T. Yordanov. Shell structure of potassium isotopes deduced from their magnetic moments. Phys. Rev. C, 90:034321, Sep 2014.

151. R. P. de Groote, J. Billowes, C. L. Binnersley, M. L. Bissell, T. E. Cocolios, T. Day Goodacre, G. J. FarooqSmith, D. V. Fedorov, K. T. Flanagan, S. Franchoo, R. F. Garcia Ruiz, Á. Koszorús, K. M. Lynch, G. Neyens, F. Nowacki, T. Otsuka, S. Rothe, H. H. Stroke, Y. Tsunoda, A. R. Vernon, K. D. A. Wendt, S. G. Wilkins, Z. Y. $\mathrm{Xu}$, and X. F. Yang. Dipole and quadrupole moments of ${ }^{73-78} \mathrm{Cu}$ as a test of the robustness of the $z=28$ shell closure near ${ }^{78} \mathrm{Ni}$. Phys. Rev. C, 96:041302, Oct 2017.

152. Akito Arima and Hisashi Horie. Configuration Mixing and Magnetic Moments of Odd Nuclei*. Progress of Theoretical Physics, 12(5):623-641, 111954.

153. Hisashi Horie and Akito Arima. Configuration mixing and quadrupole moments of odd nuclei. Phys. Rev., 99:778-785, Aug 1955. 
154. A. de Shalit and I. Talmi. Nuclear Shell Theory. Academic Press, New York, 1963.

155. C. Wraith, X.F. Yang, L. Xie, C. Babcock, J. Biero, J. Billowes, M.L. Bissell, K. Blaum, B. Cheal, L. Filippin, R.F. Garcia Ruiz, W. Gins, L.K. Grob, G. Gaigalas, M. Godefroid, C. Gorges, H. Heylen, M. Honma, P. Jnsson, S. Kaufmann, M. Kowalska, J. Krmer, S. MalbrunotEttenauer, R. Neugart, G. Neyens, W. Nrtershuser, F. Nowacki, T. Otsuka, J. Papuga, R. Snchez, Y. Tsunoda, and D.T. Yordanov. Evolution of nuclear structure in neutron-rich odd-zn isotopes and isomers. Physics Letters B, 771:385 - 391, 2017.

156. M. L. Bissell, T. Carette, K. T. Flanagan, P. Vingerhoets, J. Billowes, K. Blaum, B. Cheal, S. Fritzsche, M. Godefroid, M. Kowalska, J. Krämer, R. Neugart, G. Neyens, W. Nörtershäuser, and D. T. Yordanov. Cu charge radii reveal a weak sub-shell effect at $n=40$. Phys. Rev. C, 93:064318, Jun 2016.

157. R. Taniuchi et al. $78 \mathrm{Ni}$ revealed as a doubly magic stronghold against nuclear deformation. Nature, 569:53, 2019.

158. Tomoaki Togashi, Yusuke Tsunoda, Takaharu Otsuka, Noritaka Shimizu, and Michio Honma. Novel shape evolution in sn isotopes from magic numbers 50 to 82. Phys. Rev. Lett., 121:062501, Aug 2018.

159. A. Ekström, G. R. Jansen, K. A. Wendt, G. Hagen, T. Papenbrock, B. D. Carlsson, C. Forssén, M. Hjorth-Jensen, P. Navrátil, and W. Nazarewicz. Accurate nuclear radii and binding energies from a chiral interaction. Phys. Rev. C, 91:051301, May 2015.

160. V. Lapoux, V. Somà, C. Barbieri, H. Hergert, J. D. Holt, and S. R. Stroberg. Radii and binding energies in oxygen isotopes: A challenge for nuclear forces. Phys. Rev. Lett., 117:052501, Jul 2016.

161. S. Ettenauer et al. In preparation, 2019.

162. Á. Koszorús, X. F. Yang, J. Billowes, C. L. Binnersley, M. L. Bissell, T. E. Cocolios, G. J. Farooq-Smith, R. P. de Groote, K. T. Flanagan, S. Franchoo, R. F. Garcia Ruiz, S. Geldhof, W. Gins, A. Kanellakopoulos, K. M. Lynch, G. Neyens, H. H. Stroke, A. R. Vernon, K. D. A. Wendt, and S. G. Wilkins. Precision measurements of the charge radii of potassium isotopes. Phys. Rev. C, 100:034304, Sep 2019.

163. Á. Koszorús et al. In preparation, 2019.

164. R. De Groote et al. Precise measurement and microscopic description of charge radii of exotic copper isotopes: global trends and odd-even variations. Submitted, 2019.

165. A. Vernon et al. In preparation, 2019.

166. R.F. Garcia Ruiz et al. Laser spectroscopy of neutrondeficient sn isotopes. CERN-INTC-2016-006, INTC-P456, 2016.

167. R F Garcia Ruiz, C Gorges, M Bissell, K Blaum, W Gins, H Heylen, K Koenig, S Kaufmann, M Kowalska, J Krmer, P Lievens, S Malbrunot-Ettenauer, R Neugart, G Neyens, W Nrtershuser, D T Yordanov, and X F Yang. Development of a sensitive setup for laser spectroscopy studies of very exotic calcium isotopes. Journal of Physics G: Nuclear and Particle Physics, 44(4):044003, apr 2017.

168. R.F. Garcia Ruiz et al. Laser spectroscopy of exotic indium $(\mathrm{z}=49)$ isotopes: Approaching the $\mathrm{n}=50$ and $\mathrm{n}$ $=82$ neutron numbers. CERN-INTC-2017-025, INTCP-504, 2017.
169. Kristina D. Launey, Tomas Dytrych, and Jerry P. Draayer. Symmetry-guided large-scale shell-model theory. Progress in Particle and Nuclear Physics, 89:101 136, 2016

170. G Hagen, M Hjorth-Jensen, G R Jansen, and T Papenbrock. Emergent properties of nuclei fromab initiocoupled-cluster calculations. Physica Scripta, 91(6):063006, may 2016 .

171. T. Otsuka, Y. Tsunoda, T. Abe, N. Shimizu, and P. Van Duppen. Underlying structure of collective bands and self-organization in quantum systems. Physical Review Letters, Accepted, 2019.

172. E. Caurier, G. Martínez-Pinedo, F. Nowacki, A. Poves, and A. P. Zuker. The shell model as a unified view of nuclear structure. Rev. Mod. Phys., 77:427-488, Jun 2005.

173. Tomoaki Togashi, Yusuke Tsunoda, Takaharu Otsuka, and Noritaka Shimizu. Quantum phase transition in the shape of zr isotopes. Phys. Rev. Lett., 117:172502, Oct 2016.

174. S. Leoni, B. Fornal, N. Mărginean, M. Sferrazza, Y. Tsunoda, T. Otsuka, G. Bocchi, F. C. L. Crespi, A. Bracco, S. Aydin, M. Boromiza, D. Bucurescu, N. CieplickaOryǹczak, C. Costache, S. Călinescu, N. Florea, D. G. Ghiță, T. Glodariu, A. Ionescu, Ł.W. Iskra, M. Krzysiek, R. Mărginean, C. Mihai, R. E. Mihai, A. Mitu, A. Negreţ, C. R. Niţă, A. Olăcel, A. Oprea, S. Pascu, P. Petkov, C. Petrone, G. Porzio, A. Şerban, C. Sotty, L. Stan, I. Ştiru, L. Stroe, R. Şuvăilă, S. Toma, A. Turturică, S. Ujeniuc, and C. A. Ur. Multifaceted quadruplet of low-lying spin-zero states in ${ }^{66} \mathrm{Ni}$ : Emergence of shape isomerism in light nuclei. Phys. Rev. Lett., 118:162502, Apr 2017. 